\title{
Observation of UHE Neutrino Interactions from Outer Space
}

\author{
G. Domokos and S. Kovesi-Domokos \\ Department of Physics and Astronomy \\ The Johns Hopkins University \\ Baltimore, MD 21218
}

\begin{abstract}
The interaction of UHE neutrinos can be observed from outer space. The advantage of the proposed method is that the Earth can be used as an energy filter as well as a target. We sketch the potentials of these observations in searching for particle physics beyond the Standard Model of elementary particle interactions as well as observations of astronomical objects such as active galactic nuclei.
\end{abstract}

\section{INTRODUCTION}

It has been recognized that the observation of UHE neutrino interactions can serve a dual purpose:

- The observation of objects of high activity and high column density in the sky, (a typical example is an AGN) which otherwise are inaccessible to observation using electromagnetic waves at almost any wavelength;

- a search for particle physics not described by the current "Standard Model" of elementary particle interactions.

The first entry on our list has been widely explored in a number of articles and it has been discussed at a variety of conferences; the first meeting with the purpose of exploring all the astrophysical uses of neutrino telescopy has been the Hawaii conference [1], followed by a series of meetings on neutrino astronomy in Venice [2] and in Pylos [3].

The particle physics aspect of neutrino telescopy is somewhat less known; however, in our opinion, it is equally important. A reasonably comprehensive discussion of the particle physics uses of neutrino telescopy is contained in an article published in one of the Venice proceedings [3].

In this paper we report the results of a first exploration of the particle physics uses of a novel type of neutrino detectors: the use of orbiting detectors, such as the

1) E-mail: skd@haar.pha.jhu.edu 
Orbiting Wide angle Light collector (OWL) as well as a detector flown on a space station.

This paper is organized as follows. In the next section we outline the potential advantages of an orbiting detector used as a neutrino telescope. Section 3 contains a brief description of our first results. The final section contains a discussion and outlook.

Most of the results reported here are preliminary in nature: they will be followed up by more detailed investigations. Nevertheless, the ones reported here look exciting and, for that reason, worth discussing.

\section{II "EYE IN THE SKY"}

Conventional (?) neutrino telescopes, such as AMANDA, BAIKAL, SUPERKAMIOKANDE and the future NESTOR have the advantage that they are based on technologies which, by now, are reasonably well explored. Their basic feature is that they are capable of a good directional resolution (currently of the order of $1^{\circ}$ or so. (This is about the resolution of Galileo's telescopes: neutrino telescopy is at the age optical telescopy was in Galileo's time... ) These telescopes therefore provide a valuable tool for exploring the neutrino sky for both point like (AGN?) and diffuse sources, such as atmospheric neutrinos and neutrinos emitted by baryonic dark matter interacting with the primary cosmic radiation [4]. Likewise, on the particle physics side, such detectors are able to explore such questions whether the neutrino-nucleon cross section shows any anomaly as compared to the Standard Model [3].

What is missing is that a typical underground or underwater neutrino telescope cannot be easily made to be sensitive to the primary neutrino energy or to explore the development of a shower resulting from the interaction of the primary neutrino. To be sure, there are some solutions available to bridge the gap [5]. Most of those are, however, based on software development. It would be also desirable to have detectors which are sensitive to the primary neutrino energy directly. (The importance of this feature has been emphasized in ref. ( [3]).)

The basic idea we are here proposing is a very simple one. UHE neutrinos originating either from a point source or from a diffuse background will interact in the Earth. If the interaction takes place sufficiently close to the surface of the Earth, at least a substantial part of the shower originating from the primary interaction will develop in the atmosphere and it can be viewed by OWL or, possibly, by a future detector mounted on the space station. Due to the fact that the interaction cross section of neutrinos is energy dependent, there is a one to one correspondence between the interaction mean free path $(\mathrm{mfp})$ of the neutrino and its impact parameter with respect to the center of the Earth. (Equivalently, by an elementary exercise in geometry, the impact parameter dependence can be translated into a nadir angle dependence.)

The Earth acts as a filter: given a nadir angle and the corresponding mfp, more 
energetic neutrinos will interact deep in the Earth and the shower will die out before reaching the surface. Conversely, less energetic neutrinos either do not interact at all or they interact high in the atmosphere and there will be insufficient target thickness for the shower to develop. It is to be emphasized, of course that this are statements which hold for the average shower. Due to fluctuations in the multiplicity of the first interaction and in the shower development, one may not be able to determine shower energies on an event by event basis. (Nevertheless, simulations carried out for the Fly's Eye detector indicate that even an event by event energy determination may be feasible with a tolerable error.)

\section{AN EXAMPLE.}

In order to determine the shower development under these assumptions, our preliminary study concentrated upon the feasibility of observing a neutrino induced shower. The question one has to decide is whether the upward going shower develops over a substantial length in the atmosphere so that it can be observed. (One has to recall that an upward going shower starts in the dense part of the atmosphere, near the Earth.) For this purpose, we calculated the longitudinal development of showers in approximation A, assuming Feynman scaling. It is known that Feynman scaling is violated in hadronic interactions, due to QCD loop effects. The violation is, however, logarithmical. We took the neutrino mfp from the work of Gandhi et. al. , ref. [6]. An average hadronic cross section of $60 \mathrm{mb}$ was used, even though we find that the development of the electromagnetic component does not depend very sensitively on variations of the hadronic cross section within a reasonable range. The results were obtained (as usual) in target depth, measured in $\mathrm{g} / \mathrm{cm}^{2}$. Conversion to distances was obtained assuming an exponential atmosphere, of a scale height of $7.8 \mathrm{~km}$.

In the following Figure, we show the result of a calculation for a neutrino incident at a nadir angle of $80^{\circ}$. According to the neutrino mfp given in ref. [6] and the specifications given above, this corresponds to a neutrino energy of about $10^{9} \mathrm{GeV}$. In calculating the electromagnetic component, we assumed an initial hadron multiplicity of $\approx 50$ and an inelasticity of $\approx 0.5$. The Figure contains the longitudinal profile of the leptonic component induced by one hadron. The reason for this is that one may contemplate other multiplicities and inelasticities. In that case, the result is obtained by a simple rescaling of the ordinate. (This is a property of the diffusion approximation used here; for the present purposes, it is adequate. However, in the case of more sophisticated models, this scaling property is only approximately valid.) 
Longitudinal profile of a shower described in the text.

\section{DISCUSSION}

The example shown here suggests that one may be able to use orbiting detectors in conjunction with the Earth as a target for the detection of UHE neutrino interactions. At present, the properties of the detectors are not yet sufficiently well known and thus, no quantitative conclusions can be drawn. A more detailed calculation is needed in order to explore particle physics capabilities of the orbiting neutrino telescopes. Work along these lines is in progress.

We wish to thank the organizers of the Giant Air Shower meeting for a very stimulating conference and Bianca Monteleoni for several useful discussions on neutrino detection.

\section{REFERENCES}

1. High Energy Neutrino Astrophysics, editors V.J. Stenger et. al. World Scientific, Singapore (1992) 
2. Proceedings of the Workshops on Neutrino Telescopes, edited by M. Baldo-Ceolin. Published by the Academy of Arts, Sciences and Letters, Venice.

3. G. Domokos, B. Elliott, S. Kovesi-Domokos and S. Mrenna, in Proc. Third Workshop on Neutrino Telescopes. ( $c f$. previous reference.)

4. G. Domokos, B. Elliott and S. Kovesi-Domokos, Jour. Phys. G 19, 899 (1993).

5. B. Monteleoni, private communication.

6. R. Gandhi et. al. Astropart. Phys. 5, 81 (1996) 


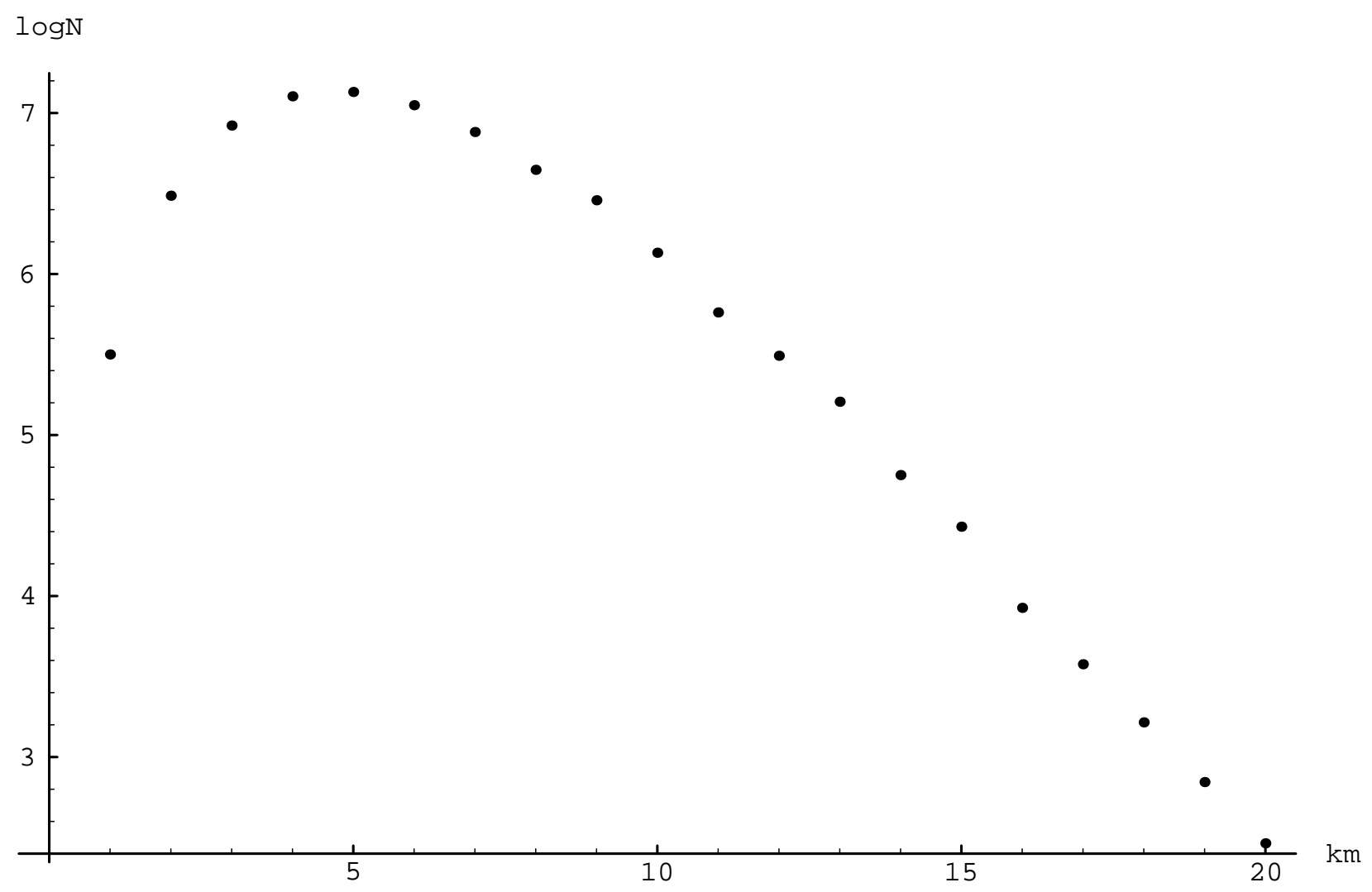

